Zwaan, L., Thijs, A., Wagner, C., Timmermans, D.R.M. Application of an evidence-based decision rule to patients with suspected pulmonary embolism. Journal of Evaluation in Clinical

\begin{tabular}{|l|l|}
\hline $\begin{array}{l}\text { Postprint } \\
\text { Version }\end{array}$ & 1.0 \\
\hline Journal website & $\underline{\text { http://onlinelibrary.wiley.com/doi/10.1111/jep.12019/abstract }}$ \\
\hline Pubmed link & $\underline{\text { http://www.ncbi.nlm.nih.gov/pubmed/23279113 }}$ \\
\hline DOI & $10.1111 /$ jep.12019 \\
\hline
\end{tabular}

This is a NIVEL certified Post Print, more info at http://www.nivel.eu

\title{
Application of an evidence-based decision rule to patients with suspected pulmonary embolism
}

\author{
Laura ZwaAn, PhD, ${ }^{1}$ Abel Thijs MD, PhD, ${ }^{2}$ Cordula Wagner, PhD $^{3}$ And DaniËLle R. \\ M. TIMMERMANS, $\mathrm{PHD}^{4}$ \\ ${ }^{1}$ Postdoctoral Researcher, \\ ${ }^{2}$ Internist, Department of Internal Medicine, VU University Medical Center, Amsterdam, The \\ Netherlands \\ ${ }^{3}$ Professor of Patient Safety, Department of Public and Occupational Health, EMGO Institute \\ for Health and Care Research, VU University Medical \\ Center, Amsterdam, The Netherlands and NIVEL, Netherlands Institute for Health Services \\ Research, Utrecht, The Netherlands \\ ${ }^{4}$ Professor of Risk Communication and Patient Decision Making, Department of Public and \\ Occupational Health, EMGO Institute for Health and Care Research, VU University Medical \\ Center, Amsterdam, The Netherlands
}

\begin{abstract}
Rationale To support doctors in diagnosing patients who are suspected to have pulmonary embolism, the Christopher evidence-based decision rule was implemented in hospitals in the Netherlands. This study examines whether the Christopher evidence-based decision rule is applied in clinical practice. In addition, doctors' considerations for not applying the decision rule are explored. Method Dyspnoea patients were included in the study prospectively. The diagnostic process of the patients with suspected pulmonary embolism, as judged by the treating doctor, was compared with the Christopher evidencebased decision rule using patient record reviews. In addition, 14 interviews were conducted with doctors who did not follow the Christopher evidence-based decision rule to obtain insights into their considerations. Results In 80 of 247 dyspnoea cases, the treating doctors suspected pulmonary embolism. The Christopher evidence-based decision rule was applied in 17 out of 80 cases. In 22 cases, more tests were performed than was suggested by the decision rule [i.e. computer- assisted tomographic angiography (CTa) or d-dimer], while in 41 cases fewer tests were performed than suggested by the decision rule. Considerations for not following the decision rule included judging another diagnosis to be more likely and not wanting to expose the patient to CTa radiation.
\end{abstract}


Zwaan, L., Thijs, A., Wagner, C., Timmermans, D.R.M. Application of an evidence-based decision rule to patients with suspected pulmonary embolism. Journal of Evaluation in Clinical

Practice: 2013, 19(4), 682-688

Conclusions The Christopher evidence-based decision rule for diagnosing pulmonary embolism was not always followed in everyday clinical practice. Doctors seem to base their diagnostic strategy on their own estimate of the likelihood of pulmonary embolism, rather than the whole decision rule. Better adherence to the decision rule could be beneficial by making doctors aware that pulmonary embolism is more likely than they initially thought. However, in a substantial number of cases, it seemed justifiable that doctors deviated from the decision rule. Therefore, further research is needed to determine the value of the Christopher evidence-based decision rule in clinical practice.

\section{INTRODUCTION}

The diagnostic process of pulmonary embolism (PE) is difficult. PE is one of the most frequently missed diagnoses [1,2]. Missing PE might have severe consequences for the patient; that is, with- holding treatment with anticoagulants could lead to higher mortality rate $[3,4]$. However, an easily applicable test that can confirm or rule out PE does not exist. The sensitivity of the d-dimer test is too low in patients with high pre-test probability to rule out $\mathrm{PE}$, and the pulmonary computer-assisted tomographic angiography (CTa) risks harming the patient, for example contrastinduced nephropathy in the short run and breast cancer in the long run [5-7]. Studies have shown that $1 \%$ of the cases having had a CTa contributed to major permanent harm to the patient, and in $0.5 \%$ of the cases potentially contributed to the patient's death [5]. There- fore, various studies have been conducted to optimize the diagnostic strategy for PE, and as a result several evidence-based decision rules have been proposed $[8,9]$. One of the most recently validated decision rules is the evidence-based decision rule of the Christopher group [10]. The Christopher decision rule differentiates between diagnostic strategies based on the a priori probability of having PE. First, using the Wells score, the patient's risk of PE can be stratified into low or high risk of having PE by examining the patient's medical history and various symptoms [11].

\section{[FIGURE 1]}

Subsequently, the Christopher decision rule states that for patients with low risk, a ddimer test needs to be performed, and when positive should be followed by a CTa. For patients with a high risk of PE, the CTa should be done immediately (see Fig. 1). In the Netherlands, the Christopher evidence-based decision rule is a general policy in hospitals. Doctors were informed about the new policy for diagnosing PE at the national symposia of their societies, and by the publication of the new guideline for diagnosing PE by the Dutch Institute for Health Care Improvement [12].

It is not known if the Christopher evidence-based decision rule is applied in everyday clinical practice. In this study, we used a database of patients presenting with dyspnoea [13], a common (but not essential) complaint in patients with PE, to examine the application of this evidence-based decision rule in everyday clinical practice. Since the decision rule does not apply to patients without suspected PE, these patients were not included in the study. The main objective of this study is (1) to determine whether doctors diagnose patients with suspected PE according to the 
Zwaan, L., Thijs, A., Wagner, C., Timmermans, D.R.M. Application of an evidence-based decision rule to patients with suspected pulmonary embolism. Journal of Evaluation in Clinical Practice: 2013, 19(4), 682-688

Christopher evidence-based decision rule and (2) to explore the considerations for not following the evidence-based decision rule.

\section{METHODS}

This study is part of a larger study in which we studied suboptimal decisions in the diagnostic reasoning process using record reviews and interviews with the treating doctors. The diagnostic reasoning process for patients presenting with shortness of breath was assessed using a prospective patient record review study [13,14]. For this sub-study, only dyspnoea patients who had suspected PE according to the treating doctor were selected. To answer the research questions, we used three data sources. Record reviews were used to determine the Wells score and to examine whether the Christopher evidence-based decision rule was applied. Doctors were asked to write down the differential diagnoses and the likelihood of those diagnoses being correct. This served two goals: it provided information (1) to determine the Wells score (together with record review) and (2) to reveal reasons why the doctors did not follow the Christopher evidence-based decision rule. To obtain insight into the reasons for not applying the Christopher evidence- based decision rule, we also analyzed several interviews with the treating doctors who did not follow the Christopher evidence- based decision rule.

\section{Patient selection}

Five acute care hospitals in the Netherlands (one university hos- pital, two tertiary teaching hospitals and two general hospitals) participated in the study. The study took place in the seven departments of internal medicine, cardiology and pulmonology. The start of the study was phased for practical reasons. The hospitals started about a month after each other, and every hospital participated during 6-8 months between May 2007 and February 2008. The treating doctors of the participating departments included all patients who (1) had dyspnoea (based on the judgement of the treating doctor) and (2) were admitted to the ward of a participating hospital department during the research period, and (3) who gave their informed consent to review their patient record. Most patients arrived through the emergency department, but patients who were transferred from another department or whose admission was planned were also eligible.

\section{[TABLE 1]}

The inclusion criteria in our study were slightly different from the inclusion criteria in the Christopher study; that is, we did not exclude patients who already were on anticoagulants, and patients who developed PE during the hospital stay were also included in our study. However, the inclusion criteria of the decision rule were chosen to obtain a homogenous patient group to test the decision rule. In everyday clinical practice, this does not happen and patients presenting with suspected PE are a heterogeneous group of patients. The decision rule, as implemented by the Dutch Institute for Healthcare Improvement (CBO), based on the Christopher study, includes all patients who have suspected $\mathrm{PE}$, and does not specifically distinguish different decision criteria for patients who are already on a dose (low or otherwise) 
Zwaan, L., Thijs, A., Wagner, C., Timmermans, D.R.M. Application of an evidence-based decision rule to patients with suspected pulmonary embolism. Journal of Evaluation in Clinical Practice: 2013, 19(4), 682-688

of anticoagulants or patients who develop symptoms of PE during their hospital stay. Thus, all patients included in our study should have been diagnosed according to the decision rule.

\section{Participating doctors}

The doctors who included the patients were residents who were employed at one of the participating hospitals. The residents were supervised by attending doctors. The doctors were aware that the diagnostic reasoning process was being studied, but they did not know that PE was being studied specifically.

\section{Record reviews}

Expert internist reviewers, who had recently retired and had at least 10 years of postgraduate work experience in internal medicine, reviewed the patient records [13]. The internist reviewers attended several training sessions and had experience with patient record reviews. According to the Christopher evidence-based decision rule, the risk of PE (high or low) is determined based on Wells score $[8,10,15]$. The Wells score consists of seven items on the patient's history and clinical findings, each item being assigned a number of points (range 1-3). The expert reviewers enquired from the patient record whether all aspects to determine the Wells score had been assessed by the treating doctors and whether they were abnormal (see Table 1). This was done retrospectively in order not to interfere in the doctor's clinical decisions. Based on this record review, in combination with the differential diagnoses as indicated by the treating doctors, the Wells score for each patient was calculated by one of the researchers (L. Z.), who was blinded to whether the patient ultimately had PE. The elements of the Wells score were generally described in the patient record, with the exception of hemoptysis which was sometimes missing. Since hemoptysis is an important factor, we assume that in cases showing deviance, this was mentioned in the patient record. There- fore, if it was not described in the patient record, we assumed the patient did not have hemoptysis.

Based on the total Wells score, the patient's risk of PE can be determined. The Christopher evidence-based decision rule divides the Wells score into two categories: low risk and high risk of PE with a cut-off value of 4 (:S4 represents low risk, >4 represents high risk) $[10,15]$. When the Wells score is :S4, a d-dimer test needs to be performed. If the d-dimer is elevated ( $500 \mathrm{ng} \mathrm{mL-1}$ is the currently used cut-off value), a CTa will confirm or rule out PE. For patients with a Wells score $>4$, a CTa needs to be performed immediately to confirm or rule out PE (see Fig. 1). These diagnostic steps of the Christopher evidence-based decision rule were also obtained from the record review, that is the application of the d-dimer and the CTa.

\section{Differential diagnoses}

We asked the treating doctors to note down their differential diagnoses after the first diagnostic steps (history taking, physical examination and results of laboratory tests). The differential diagnoses were written down in order of likelihood (1 to maximum of 5 diagnoses), and the numerical likelihood of each of the diagnoses was indicated on a visual analogue scale of 1-10 (1 being very unlikely, 10 being very likely). 
Zwaan, L., Thijs, A., Wagner, C., Timmermans, D.R.M. Application of an evidence-based

decision rule to patients with suspected pulmonary embolism. Journal of Evaluation in Clinical

\section{Interviews}

Treating doctors were interviewed about their considerations in the diagnostic process. We selected 14 cases in which the doctors performed fewer diagnostic tests than was suggested by the decision rule to diagnose PE. The interviews consisted of simple questions such as the following: 'The elevated d-dimer was not followed by a $\mathrm{CTa}$, could you explain why not?' or 'PE was a possible diagnosis, why did you decide not to conduct any further tests to establish or rule out PE?' The answers of the treating doctors were noted down and clustered by a researcher (L. Z.).

\section{Statistical analysis}

Descriptive statistics were used for the quantitative analyses. The interviews were analyzed by clustering the answers into categories, making sure all answers were represented in the themes.

\section{Confidentiality and ethical approval}

The review board of the VU Medical Center approved of the research protocol, and all participating hospitals granted approval to participate. The internist reviewers and researchers involved in the data collection signed a confidentiality agreement to preserve the secrecy of the data. Patients included in the study gave informed consent to review their patient record.

\section{[TABLE 2]}

\section{RESULTS}

\section{Application of the Christopher evidence-based decision rule}

A total of 261 patients were included in the study, of which 14 were excluded because their patient records could not be obtained. For 80 out of 247 dyspnoea patients, the doctors indicated PE in the differential diagnoses (see Table 2 for patient characteristics). Fifty-six patients had a low risk of PE (Wells :S4), and 24 patients had a high risk of PE (Wells $>4$ ). The doctors diagnosed PE according to the Christopher evidence-based decision rule in 21\% [17 patients, 95\% confidence interval (CI): 13.7-31.4\%]. In 51\% (41 patients, 95\% CI: 40.5-61.9\%), fewer or no diagnostic tests were used, which could potentially lead to a missed PE. In 28\% (22 patients, 95\% CI: 18.9-38.4\%), more diagnostic tests were conducted than was suggested by the Christopher decision rule.

Of the patients with a low risk (Wells :S4) of PE, in 63\% (35 out of 56, 95\% CI: 49.5-74.0\%) of the cases, no diagnostic tests or insufficient diagnostic tests were performed (see Fig. 2). Further- more, out of the eight cases in which the d-dimer was not elevated, the doctors applied CTa in four cases. In 10 cases, a CTa was performed immediately without a prior d-dimer.

Of the patients with a high risk of PE (Wells score $>4$ ), for $21 \%-5$ of 24 patients (95\% CI: 9.2-40.5\%) - no further diagnostic tests were performed (see Fig. 3). In one patient, an elevated d-dimer was not followed by a CTa, and PE was initially missed. In 13 cases, the doctor performed a d-dimer, while the Christopher evidence- 
Zwaan, L., Thijs, A., Wagner, C., Timmermans, D.R.M. Application of an evidence-based decision rule to patients with suspected pulmonary embolism. Journal of Evaluation in Clinical Practice: 2013, 19(4), 682-688

based decision rule recommends an immediate CTa. Those cases showed an elevated d-dimer in $92 \%, 12$ out of 13 patients.

\section{Likelihood of PE in differential diagnoses}

Doctors were significantly more likely to refrain from further diagnostic tests to diagnose or rule out PE when they considered PE to be less likely than other diagnoses [i.e. the rank of $\mathrm{PE}$ was lower in the differential diagnoses $(\mathrm{X} 2=13.3, \mathrm{P}<$ 0.0001)]. The likelihood ratings of PE of the cases with a Wells score :S4 were low. When the Wells score was $>4$, the likelihood ratings were high, except for cases in which no further diagnostics were per- formed, in which case they were significantly lower compared with cases in which diagnostic tests were performed $(\mathrm{t}=3.75, \mathrm{P}=$ 0.001) (Table 3).

\section{Reasons for not applying the Christopher evidence-based decision rule}

We conducted interviews in cases in which PE might have been missed. This involved cases where no diagnostic tests were done and cases without an adequate follow-up on an elevated d-dimer.

\section{No further diagnostic tests}

When the doctor did not perform further diagnostic tests, the following three clusters of reasons were mentioned:

- An alternative diagnosis was more likely than PE; therefore, PE was not further examined.

'I thought pneumonia was more likely considering the patient's symptoms and therefore I did not perform diagnostic tests, i.e. d-dimer'.

'There was a low suspicion of DVT or PE and the patient's main symptom was pain in the thorax with an unclear cause. It was a deliberate choice not to perform diagnostic tests for DVT and PE, mainly because the patient would get anticoagulants for a different reason anyway'.

\section{No adequate follow-up after elevated D-dimer value}

When the doctor did not follow up on an elevated d-dimer value (which would require a CTa), two clusters of reasons were mentioned:

- Assuming the high d-dimer value was due to other reasons.

'The Wells score was low and I expected the d-dimer was elevated because of a high C-reactive protein, therefore I did not perform a CTa'.

- Not wanting to expose patients to unnecessary tests while anti- coagulants were already prescribed for another disease.

'The patient already got anticoagulants for other reasons and therefore I did not perform a CTa which can also be harmful for the patient'.

\section{DISCUSSION}

Our results show that doctors do not always use the Christopher evidence-based decision rule when diagnosing PE, although it is the general guideline in Dutch hospitals. The Christopher evidence-based decision rule was followed in only $21 \%$ of 
Zwaan, L., Thijs, A., Wagner, C., Timmermans, D.R.M. Application of an evidence-based decision rule to patients with suspected pulmonary embolism. Journal of Evaluation in Clinical Practice: 2013, 19(4), 682-688

the patients with suspected PE, while in $79 \%$ of the patients it was not followed. Reasons for not applying the Christopher decision rule were, for example, considering the likelihood of $\mathrm{PE}$ to be too low to warrant performing more tests. Our study has several limitations. First, due to the clinical setting and the record review, the Wells score might be an under- estimation for some cases. Sometimes, the treating doctor did not examine all elements of the Wells score (i.e. hemoptysis) or did not write them down in the patient record. This means that some of the patients with a Wells score of :S4 actually could have had a Wells score of $>4$. Therefore, the decision to perform an immediate CTa might have been correct for some of the cases. Specifically, in our study, two out of ten patients with low risk (Wells :S4) who got an immediate CTa had a PE. This figure was lower in the original study of the Christopher group [10]. Second, we examined only the cases in which the doctor considered PE as a possible diagnosis after the first examination of the patient. Cases of patients with a missed PE because the doctor did not think of PE in the first place were not examined. This is not part of the decision rule, and therefore was outside the scope of this article. Third, we only interviewed doctors who conducted fewer diagnostic tests than was recommended by the decision rule. Last, since the PE diag- nosis was determined by record review, we do not have a complete overview of the patients who actually had PE. Some of the patients died, either in the hospital or at home after being discharged, and no autopsy was performed. Therefore, results on the prevalence of $\mathrm{PE}$ and the number of PE diagnoses that were missed might be an underestimation.

The doctors were familiar with the Christopher evidence-based decision rule and the Wells score. However, they did not follow the decision rule in the majority of the cases. This demonstrates that developing and implementing a decision rule does not mean that it is always used in everyday practice, and an evaluation of imple- mented decision rules in clinical practice is desirable.

Following the Christopher decision rule does not always lead to the desired outcome. Specifically, the decision rule is based on data from a large number of patients, and is therefore not applica- ble to all individual cases. Therefore, adherence to the rule in all circumstances would be incorrect. When circumstances indicate that deviating from the rule is desirable, this should be possible. Nevertheless, the Christopher decision rule is considered to be a valid decision rule, and therefore should be applicable in the majority of the cases $[10,16]$.

In this study, we found that the rule was followed in a minority of patients with suspected PE. Research shows that doctors can correctly determine in which cases they need to deviate from a guideline [17]. This probably also accounts to some extent for this study. The most frequent deviations from the decision rule are unlikely to cause harm to the patient (i.e. no diagnostics in patients with a low Wells score, and an unnecessary d-dimer in patients with a high Wells score). Furthermore, in one case, not adhering to the decision rule revealed a PE.

However, of some deviations, it is less clear whether they are justifiable. These involve the deviations from the decision rule that may cause harm to the patient: (1) unnecessary CTas in patients with a low risk of PE, and (2) no diagnostic assessment of PE in patients with a high risk of PE. Based on this study, it is difficult to determine whether the CTas conducted in the group of patients with a low Wells score was incorrect because of the potential underestimation of the Wells score. 
Zwaan, L., Thijs, A., Wagner, C., Timmermans, D.R.M. Application of an evidence-based decision rule to patients with suspected pulmonary embolism. Journal of Evaluation in Clinical
Practice: $2013,19(4), 682-688$

However, we can provide some insights into reasons why no further diagnostics were conducted in cases with high Wells score. Doctors seemed to base their diagnostic strategy on their own estimate of the likelihood of PE, rather than the whole decision rule. When their estimate of the likelihood of PE was low (but the Wells score was high), they did not conduct further diagnostic testing (see Table 3). Although the explanations they provided seem legitimate for some cases, it is not always clear whether in all cases it was justifiable to refrain from further diagnostic assessment of PE. Specifically, when a patient has PE, a specific dose and duration of anticoagulants is needed, and the dose and/or duration provided for another disease might be insufficient. Also, when a d-dimer is suspected to be elevated due to comorbidity, this does not mean that the patient does not have PE [18]. In fact, PE often co-occurs with other diseases, such as asthma and COPD, and has a higher mortality rate, getting missed more often in those cases [18,19]. A missed PE is still one of the most frequently missed diagnoses, and consequences of a missed PE can be severe $[2,20]$. Therefore, consideration of PE as co-morbidity is important.

Overall, the results of this study raise the question whether the decision rule is valuable in clinical practice. It is, therefore, impor- tant that further research is initiated to determine whether the Christopher decision rule applies to a sufficient number of cases in clinical practice. However, in some cases, the doctors base their diagnostic strategy too strongly on their own estimate of the like- lihood of PE. The Christopher evidence-based decision rule could be helpful to make doctors aware that PE is more likely than they expected.

\section{CONCLUSIONS}

The Christopher evidence-based decision rule based on the Wells score to diagnose $\mathrm{PE}$ is often not followed in everyday clinical practice even though the use of the prediction rule is propagated nationally in the national guideline for diagnosing $\mathrm{PE}$. In some cases, better adherence to the decision rule could be beneficial in making doctors aware that PE is more likely than they expected. On the other hand, doctors seemed to correctly deviate from the decision rule in a substantial number of cases. Therefore, further research is needed to determine the value of the Christopher evidence-based decision rule in clinical practice.

\section{Acknowledgements and funding}

The authors would like to thank everyone who contributed to the study: S.

Lubberding, MSc, for her help with the data gathering, and the doctors who reviewed the patient records, H. A. P. Ass- cheman, MD, PhD; W. W. Meijer, MD; K. J. Roozendaal, MD, PhD; and J. Silberbusch, MD, PhD. We would also like to thank the hospitals and their staff for including the patients in the study and for facilitating the patient records.

The study was initiated and supported by the Dutch Society of Medical Specialists (in Dutch: Orde van Medisch Specialisten), with financial support from the Dutch Ministry of Health, Welfare and Sport. The study protocol was reviewed and approved by the scientific committee of the EMGO Institute and by the ethical review board of the VU University Medical Center. 
Zwaan, L., Thijs, A., Wagner, C., Timmermans, D.R.M. Application of an evidence-based decision rule to patients with suspected pulmonary embolism. Journal of Evaluation in Clinical Practice: 2013, 19(4), 682-688

\section{REFERENCES}

1. Aalten, C., Samson, M. \& Jansen, P. (2006) Diagnostic errors; the need to have autopsies. The Netherlands Journal of Medicine, 64 (6), 186-190.

2. Zwaan, L., De Bruijne, M. C., Wagner, C., Thijs, A., Smits, M., Van der Wal, G. \& Timmermans, D. R. M. (2010) A record review on the incidence, consequences and causes of diagnostic adverse events. Archives of Internal Medicine, 170 (12), 1015-1021.

3. Barritt, D. W. \& Jordan, S. C. (1960) Anticoagulant drugs in the treatment of pulmonary embolism. A controlled trial. Lancet, 1 (7138), 1309-1312.

4. Carson, J. L., Kelley, M. A., Duff, A., et al. (1992) The clinical course of pulmonary embolism. The New England Journal of Medicine, 326 (19), 1240-1245.

5. Stein, P. D., Athanasoulis, C., Alavi, A., Greenspan, R. H., Hales, C. A., Saltzman, H. A., Vreim, C. E., Terrin, M. L. \& Weg, J. G. (1992) Complications and validity of pulmonary angiography in acute pul- monary embolism. Circulation, 85 (2), 462-468.

6. Smith-Bindman, R. (2010) Is computed tomography safe? The New England Journal of Medicine, 363 (1), 1-4.

7. Parker, M. S., Hui, F. K., Camacho, M. A., Chung, J. K., Broga, D. W. \& Sethi, N. N. (2005) Female breast radiation exposure during CT pulmonary angiography. AJR. American Journal of Roentgenology, 185, 1228-1233.

8. Wells, P., Grinsberg, J., Anderson, D., et al. (1998) Use of a clinical model for safe management of patients with suspected pulmonary embolism. Annals of Internal Medicine, 129, 997-1005.

9. Wicki, J., Perneger, T., Junod, A., Bounameaux, H. \& Perrier, A. (2001) Assessing clinical probability of pulmonary embolism in the emergency ward. Archives of Internal Medicine, 8 (161), 92- 97.

10. The Christopher Writing Group (2006) Effectiveness of managing suspected pulmonary using an algorithm combining clinical probabil- ity, d-dimer testing and computed tomography. JAMA: The Journal of the American Medical Association, 295, 172-179.

11. Wells, P. S., Anderson, D. R., Rodger, M., et al. (2000) Derivation of a simple clinical model to categorize patients probability of pulmonary embolism: increasing the models utility with the SimpliRED d-dimer. Thrombosis and Haemostasis, 83 (3), 416-420.

12. Dutch Institute for Health Care Improvement (CBO) (2008) Richtlijn Diagnostiek, preventie en behandeling van veneuze trombo-embolie en secundaire preventie van arteriele trombose. Alphen aan den Rijn: Van Zuiden Communications B.V.

13. Zwaan, L., Thijs, A., Wagner, C., Van der Wal, G. \& Timmermans, D. R. M. (2009) Design of a study on suboptimal cognitive acts in the diagnostic process, the effect on patient outcomes and the influence of workload, fatigue and experience of physicians. BMC Health Services Research, 9, 65. doi: 10.1186/1472-6963-9-65.

14. Zwaan, L., Thijs, A., Wagner, C., Van der Wal, G. \& Timmermans, D. (2012) Relating faults in diagnostic reasoning with diagnostic errors and patient harm. Academic Medicine, 87 (2), 149-56.

15. Wells, P. S., Anderson, D. R., Rodger, M., Stiell, I., Dreyer, J. F., Barnes, D., Forgie, M., Kovacs, G., Ward, J. \& Kovacs, M. (2001) Excluding pulmonary embolism at the bedside without diagnostic imaging: management of patients with suspected pulmonary embolism presenting to the emergency department by using a simple clinical model and d-dimer. Annals of Internal Medicine, 135, 98-107.

16. Geersing, G., Erkens, P., Lucassen, W., et al. (2012) Safe exclusion of pulmonary embolism using the Wells rule and qualitative d-dimer testing in primary care: prospective cohort study. BMJ (Clinical Research Ed.), 345. doi: 10.1136/bmj.e6564.

17. Persell, S., Dolan, N., Friesema, E., Thompson, J., Kaiser, D. \& Baker, D. (2010) Frequency of inappropriate medical exceptions to quality measures. Annals of Internal Medicine, 152, 225-231.

18. Pidenda, L. A., Hathwar, V. S. \& Grand, B. J. (2001) Clinical suspicion on fatal pulmonary embolism. Chest, 120, 791-795.

19. Carson, J., Terrin, M., Duff, A. \& Kelley, M. A. (1996) Pulmonary embolism and mortality in patients with COPD. Chest, 110 (5), 1135-1136.

20. Schiff, G. D., Hasan, O., Kim, S., et al. (2009) Diagnostic error in medicine: analysis of 583 physician-reported errors. Archives of Inter- nal Medicine, 169 (20), 1881-1887. 
Zwaan, L., Thijs, A., Wagner, C., Timmermans, D.R.M. Application of an evidence-based decision rule to patients with suspected pulmonary embolism. Journal of Evaluation in Clinical Practice: 2013, 19(4), 682-688

\section{TABLES}

Figure 1 The Christopher evidence-based decision rules for diagnosing pulmonary embolism. CTa, computer-assisted tomographic angiography; DVT, deep venous thrombosis; PE, pulmonary embolism.

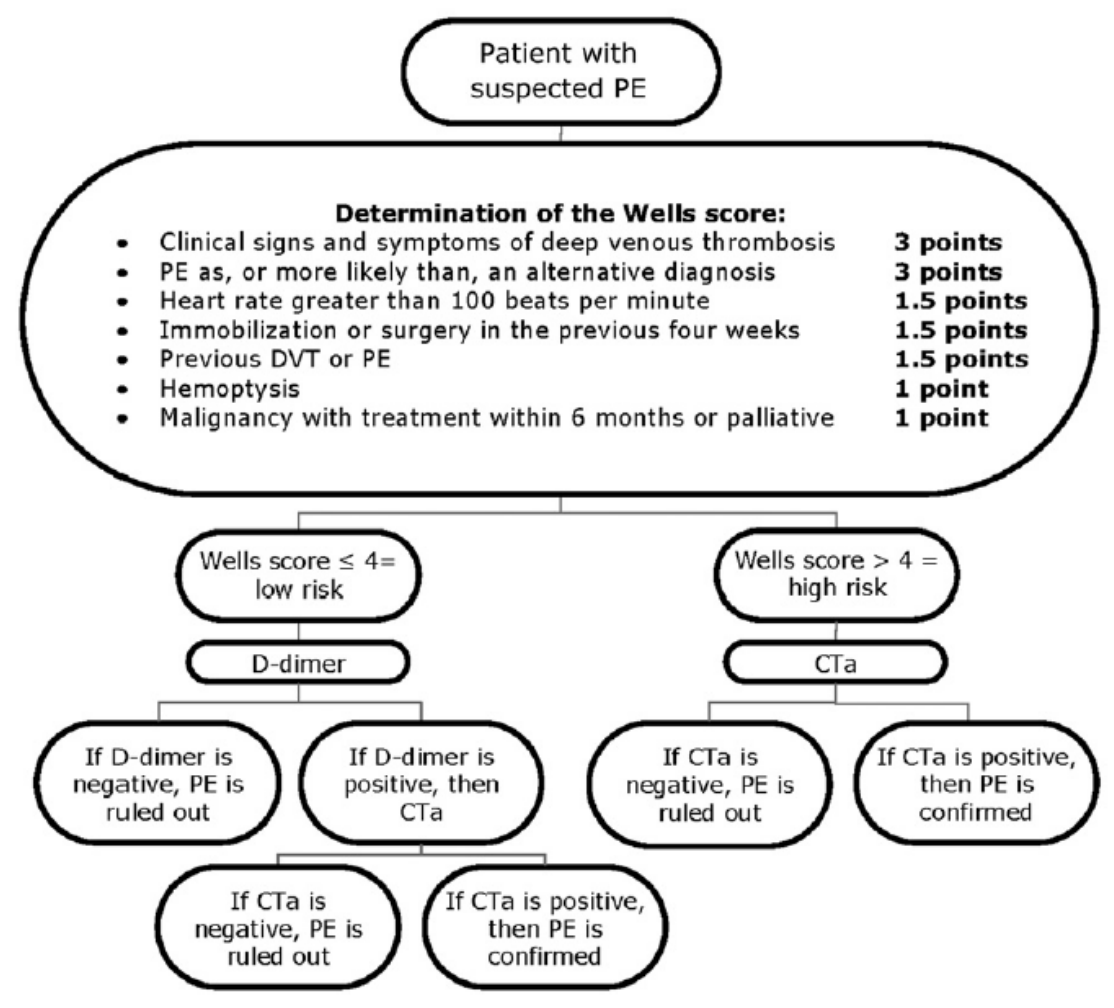


Zwaan, L., Thijs, A., Wagner, C., Timmermans, D.R.M. Application of an evidence-based decision rule to patients with suspected pulmonary embolism. Journal of Evaluation in Clinical

Practice: 2013, 19(4), 682-688

Table 1 The questions of the record review used to determine Wells score [11]

\begin{tabular}{|c|c|}
\hline Wells score & Questions in record review \\
\hline Clinical signs and symptoms of DVT & $\begin{array}{l}\text { - Colour of lower leg } \\
\text { - Symmetry of lower leg } \\
\text { - Pain in lower leg }\end{array}$ \\
\hline $\begin{array}{l}\text { PE as, or more likely, than an alternative } \\
\text { diagnosis }\end{array}$ & $\begin{array}{l}\text { - The likelihood of the differential diagnoses was indicated after the first examination of the } \\
\text { patient. When the value of PE was highest or the same as the highest alternative, this item } \\
\text { of the Wells score was considered to be true. }\end{array}$ \\
\hline Heart rate greater than 100 beats per minute & - Was the pulse rate checked/abnormal? \\
\hline $\begin{array}{l}\text { Immobilization or surgery in the previous } \\
\text { four weeks }\end{array}$ & $\begin{array}{l}\text { - When a recent surgery or immobilization was mentioned in the description of co-morbidity } \\
\text { or recent history } \\
\text { - When the patient was relocated to the participating department after surgery } \\
\text { - When immobilization was mentioned as a reason for hospital admission }\end{array}$ \\
\hline Previous DVT or PE & - When a DVT or PE was mentioned in the description of co-morbidity or recent history \\
\hline $\begin{array}{l}\text { Malignancy (with treatment within six months } \\
\text { or palliative) }\end{array}$ & - When a malignancy was mentioned in the description of co-morbidity or recent history \\
\hline
\end{tabular}

DVT, deep venous thrombosis; PE, pulmonary embolism.

Table 2 Characteristics of patients with suspected pulmonary embolism $(n=80)$

\begin{tabular}{lc}
\hline Average age (SD) & $67.8(14.3)$ \\
Male sex & $33(41.3 \%)$ \\
Final diagnosis & $14(18 \%)$ \\
Pulmonary embolism & $16(20 \%)$ \\
Chronic obstructive pulmonary disease/bronchitis/ & \\
$\quad$ asthma & $12(15 \%)$ \\
Pneumonia & $11(14 \%)$ \\
Heart failure & $6(8 \%)$ \\
Malignancy & $21(25 \%)$ \\
Others (e.g. ischaemic heart disease, cholecystitis, & \\
$\quad$ pneumothorax, viral infection, hyperventilation, & \\
$\quad$ bronchiolitis) & $30(38 \%)$ \\
Relevant co-morbidity (can be more than one diagnosis & \\
$\quad$ per patient) & $4(9 \%)$ \\
Malignancy & $4(9 \%)$ \\
Chronic obstructive pulmonary disease/bronchitis/ & \\
$\quad$ asthma & $3(7 \%)$ \\
Pneumonia & $3(7 \%)$ \\
Heart failure & $3(7 \%)$ \\
Hypoglycaemia & $2(5 \%)$ \\
Renal insufficiency & $2(5 \%)$ \\
Ischaemic heart disease without heart failure & $1(2 \%)$ \\
Pulmonary embolism & $22(49 \%)$ \\
Others (e.g. aorta valve stenosis, viral infection, & $8(11)$ \\
$\quad$ lung fibrosis, liver failure, HIV) &
\end{tabular}


Zwaan, L., Thijs, A., Wagner, C., Timmermans, D.R.M. Application of an evidence-based decision rule to patients with suspected pulmonary embolism. Journal of Evaluation in Clinical Practice: 2013, 19(4), 682-688

Figure 2 Diagnostic steps as performed by the doctors for patients with a Wells score _4. CTa, computer-assisted tomographic angiography; PE, pulmonary embolism.

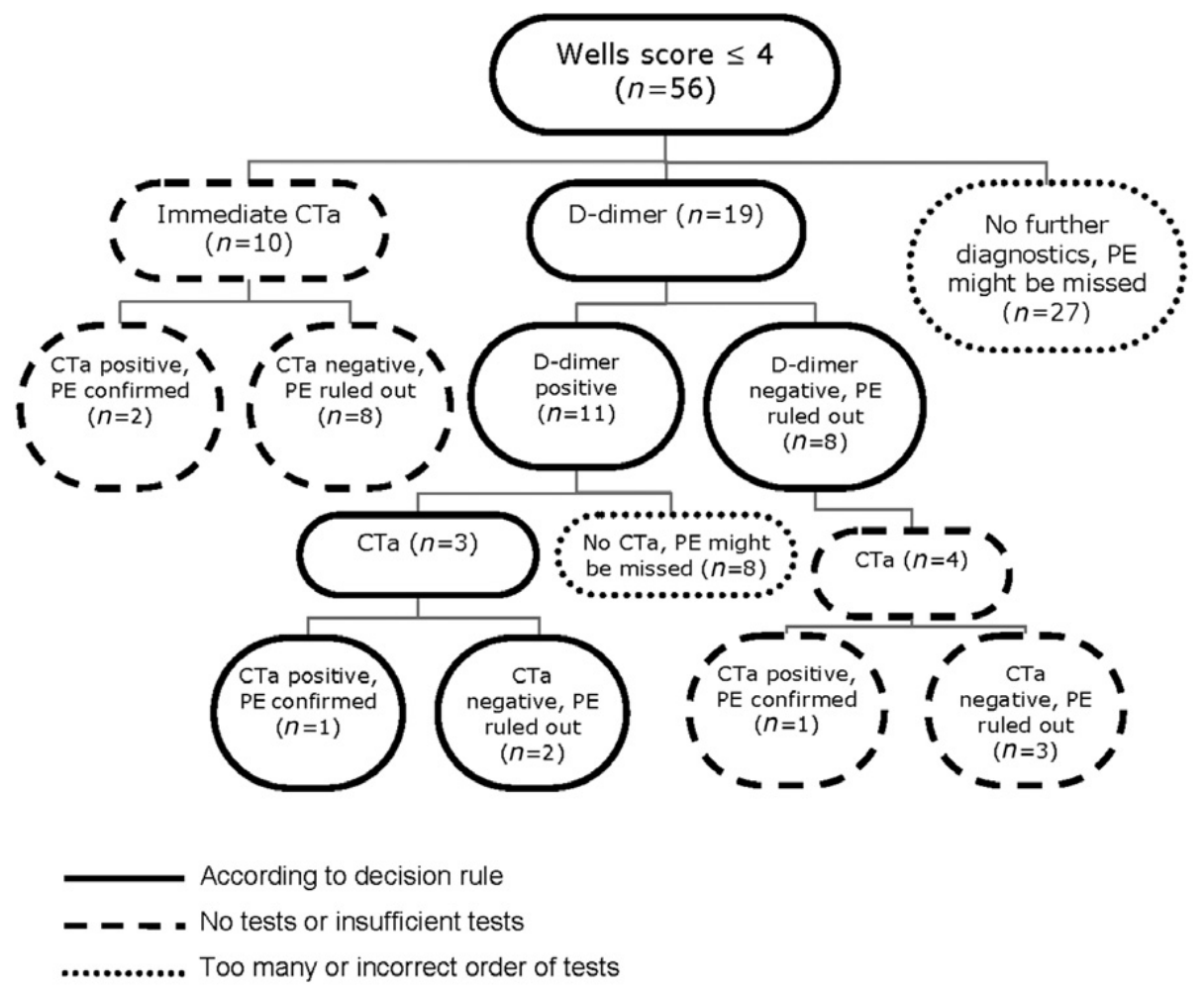

Figure 3 Diagnostic steps as performed by the doctors for patients with a Wells score $>4$. CTa, computer-assisted tomographic angiography; PE, pulmonary embolism.

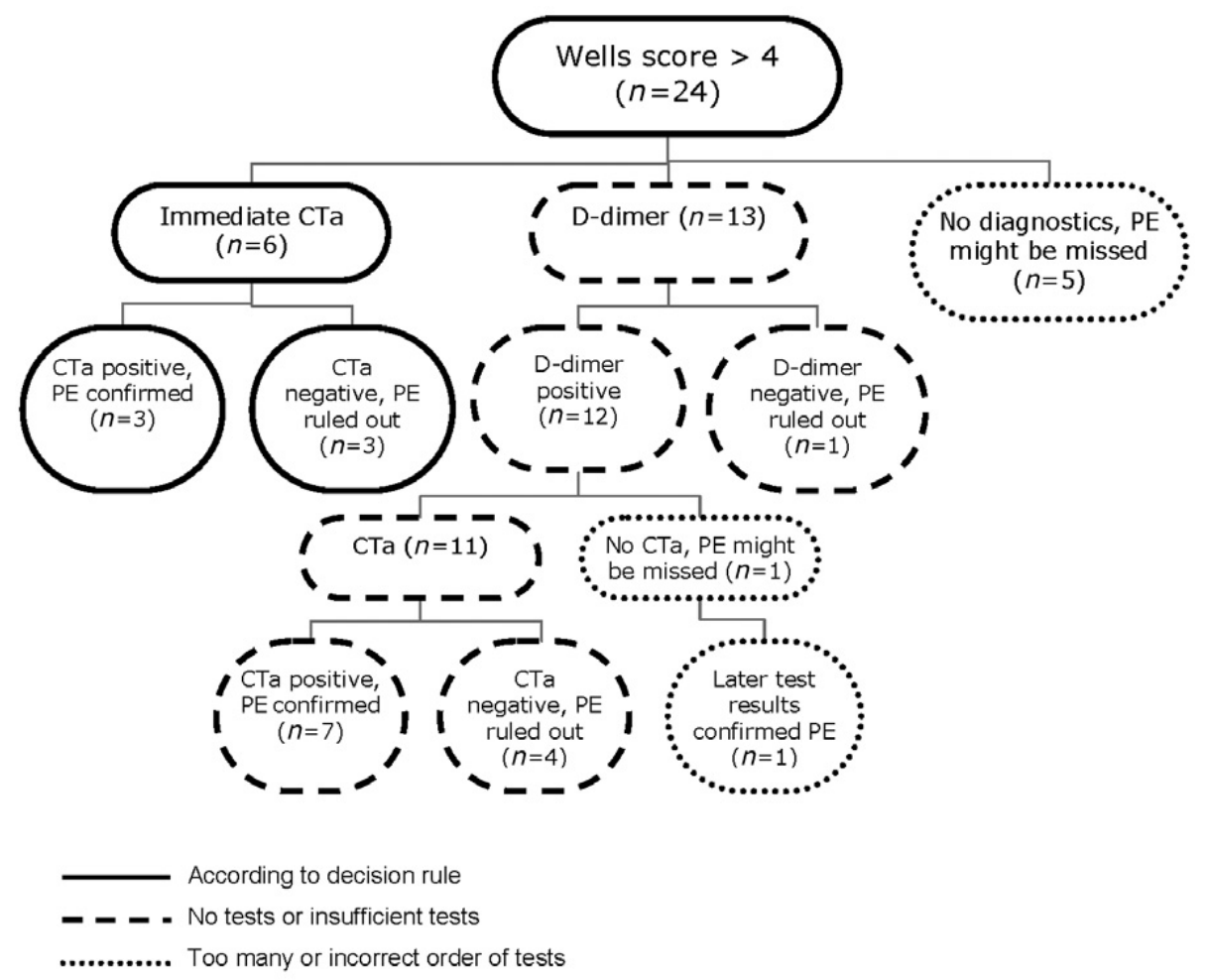


Zwaan, L., Thijs, A., Wagner, C., Timmermans, D.R.M. Application of an evidence-based decision rule to patients with suspected pulmonary embolism. Journal of Evaluation in Clinical Practice: 2013, 19(4), 682-688

Table 3 Average Wells score and doctor's considerations for the different diagnostic strategies

\begin{tabular}{lllll}
\hline & & & $\begin{array}{l}\text { Rank of PE in DD, } \\
\text { mean (range) }\end{array}$ & $\begin{array}{l}\text { Likelihood of PE in DD, } \\
\text { mean (range) }\end{array}$ \\
\hline Wells $\leq 4$ & No diagnostic tests & 28 & $3.0(1-5)$ & $3.7(1-9)$ \\
& D-dimer first & 18 & $2.8(1-5)$ & $3.8(1-8)$ \\
& Immediate CTa & 10 & $2.8(1-4)$ & $4.1(2-10)$ \\
Wells $>4$ & No diagnostics tests & 5 & $2.8(2-4)$ & $4.0(1-7)$ \\
& D-dimer first & 13 & $1.2(1-3)$ & $8.3(2-10)$ \\
& Immediate CTa & 6 & $1.3(1-2)$ & $8.3(7-10)$ \\
\hline
\end{tabular}

$\mathrm{CTa}$, computer-assisted tomographic angiography; DD, differential diagnosis; PE, pulmonary embolism. 